\title{
Identification of tyrosine 806 as a molecular determinant of RET kinase sensitivity to ZD6474
}

\author{
Francesca Carlomagno, Teresa Guida, Suresh Anaganti, Livia Provitera, \\ Svend Kjaer ${ }^{1}$, Neil Q McDonald ${ }^{1}$, Anderson J Ryan ${ }^{2}$ and Massimo Santoro
}

Istituto di Endocrinologia ed Oncologia Sperimentale del CNR, c/o Dipartimento di Biologia e Patologia Cellulare e Molecolare, Università di Napoli Federico II, via S. Pansini 5, 80131 Naples, Italy

${ }^{1}$ Structural Biology Laboratory, London Research Institute, Cancer Research UK, London, UK

${ }^{2}$ Cancer Discovery, Astra Zeneca, Mereside, Alderley Park, Macclesfield, Cheshire, UK

(Correspondence should be addressed to M Santoro; Email: masantor@unina.it)

\begin{abstract}
ZD6474 (vandetanib, Zactima, Astra Zeneca) is an anilinoquinazoline used to target the receptor tyrosine kinase RET in familial and sporadic thyroid carcinoma $\left(\mathrm{IC}_{50}: 100 \mathrm{nM}\right)$. The aim of this study was to identify molecular determinants of RET sensitivity to ZD6474. Here, we show that mutation of RET tyrosine 806 to cysteine (Y806C) induced RET kinase resistance to ZD6474 $\left(\mathrm{IC}_{50}: 933 \mathrm{nM}\right)$. Y806 maps close to the gate-keeper position at the RET kinase nucleotide-binding pocket. Although tyrosine 806 is a RET auto-phosphorylation site, its substitution to phenylalanine (Y806F) did not markedly affect RET susceptibility to ZD6474 ( $\left.\mathrm{IC}_{50}: 87 \mathrm{nM}\right)$, suggesting that phosphorylation of $\mathrm{Y} 806$ is not required for compound binding. Accordingly, the introduction of a phosphomimetic residue (Y806E) also caused resistance to ZD6474, albeit of a lesser degree $\left(\mathrm{IC}_{50}: 512 \mathrm{nM}\right)$ than the cysteine mutation. Y806C/E RET mutants were also resistant to ZD6474 with respect to intracellular signalling and activation of an AP1-responsive promoter. We conclude that Y806 is a molecular determinant of RET sensitivity to ZD6474. Y806C is a natural RET mutation identified in a patient affected by multiple endocrine neoplasia type $2 \mathrm{~B}$. Based on its rare occurrence, it is unlikely that Y806C will be a frequent cause of refractoriness to ZD6474; however, it may be envisaged that mutations at this site can mediate secondary resistance formation in patients treated with the compound.
\end{abstract}

Endocrine-Related Cancer (2009) 16 233-241

\section{Introduction}

Small molecule tyrosine kinase inhibitors (TKI) are effective in a variety of human cancers driven by the activation of specific tyrosine kinases (Krause \& Van Etten 2005, Baselga 2006). A paradigmatic example is imatinib mesylate (Gleevec, STI571), an ATPcompetitive inhibitor of BCR-ABL, KIT and PDGFR kinases. Imatinib is used for the treatment of chronic myeloid leukaemia (CML) patients harbouring the BCR-ABL translocation and of gastrointestinal stromal tumour patients harbouring KIT or PDGFR $\alpha$ mutations (Sherbenou \& Druker 2007).

Although most CML cases initially respond to imatinib, relapses are frequent, particularly in patients with advanced disease. Point mutations within the kinase domain of BCR-ABL are the most frequent causes of resistance. Such mutations can either remove residues that are critical for drug-kinase interaction, or create steric hindrance or display an allosteric effect, preventing the kinase from adopting the correct conformation for drug binding (Daub et al. 2004, Nardi et al. 2004, Weisberg et al. 2007). The best described resistance-associated mutation affects T315, the so called 'gate-keeper' site. T315 is located at the base of the ATP-binding pocket, where also the drug binds. Amino acid changes at the gate-keeper site are able to confer resistance not only to imatinib but also to other BCR-ABL inhibitors, such as dasatinib and nilotinib (Kantarjian et al. 2006, Talpaz et al. 2006). Moreover, mutation of the corresponding residue has 
been linked to the resistance of other tyrosine kinases to their specific TKI (Pao et al. 2005).

How resistance to kinase inhibitors develops in patients is being understood. The short lag of time in which resistance develops in CML patients has suggested that resistance-causing mutations are present before treatment in a few tumour cells, in cis with the oncogenic alteration, and are maintained in the tumour cell population because they increase the relative fitness of the mutated cell clones. Tumour cells harbouring the resistance-causing mutation, in turn, could be positively selected upon treatment with imatinib (secondary resistance; Sherbenou \& Druker 2007). In other cases, the initial oncogenic mutation per se is refractory to the drug (primary resistance). For instance, tumour-associated KIT and PDGFR $\alpha$ variants displaying gain-of-function mutations in the kinase activation loop (D816 in KIT and D842 in PDGFR $\alpha$ ) are refractory to imatinib, and patients harbouring these mutations respond poorly to imatinib (Corless et al. 2005). Similarly, a germline EGFR mutation $(\mathrm{T} 790 \mathrm{M})$ found in rare families predisposed to lung cancer not only activates the oncogenic potential of EGFR but also causes resistance to the EGFR TKIs gefitinib and erlotinib (Bell et al. 2005). An understanding of the molecular basis of drug sensitivity and resistance is required to interpret results of clinical trials with TKIs, to select patients for treatment and to design strategies aimed at circumventing resistance formation (Kantarjian et al. 2006, O'Hare et al. 2006, Talpaz et al. 2006, Shah et al. 2007).

RET receptor tyrosine kinase is frequently activated in thyroid tumours (Santoro \& Carlomagno 2006). Germline point mutations affecting the extracellular or kinase domains of RET are associated to the autosomal dominant multiple endocrine neoplasia type 2 (MEN 2) syndromes (MEN 2A, MEN 2B, familial medullary thyroid cancer), characterized by the occurrence of medullary thyroid carcinoma (MTC), pheochromocytoma, parathyroid adenoma, and ganglioneuromatosis of the gut. Somatic RET point mutations are found in 30-40\% of sporadic MTC cases (Marx 2005, Schlumberger et al. 2008). Most of the MEN 2/MTC-associated RET mutations either target extracellular cysteine residues (typically in MEN 2A), or the methionine 918 (typically in MEN 2B) or few other residues (E768, L790, Y791, V804, S891) in the RET kinase domain. Some patients harbour more rare mutations in other codons or small insertions/deletions (Santoro \& Carlomagno 2006). Moreover, chromosomal rearrangements involving the RET kinase encoding domain (chimeric RET/papillary thyroid carcinoma (PTC) oncogenes) are found in (PTC;
Santoro \& Carlomagno 2006). Both MEN 2/MTCassociated point mutations and RET/PTC rearrangements switch on the enzymatic function of RET in a ligand-independent manner (Santoro \& Carlomagno 2006). Whereas adjuvant radiometabolic therapy with ${ }^{131}$ I is effective after surgery for PTC, MTC does not respond to conventional radiotherapy or chemotherapy, and early surgery remains the only treatment for this tumour (Schlumberger et al. 2008).

ZD6474 (Zactima, vandetanib) is an orally bioavailable anilinoquinazoline with strong inhibiting activity against VEGFR-2 $\left(\mathrm{IC}_{50}=40 \mathrm{nM}\right)$ and EGFR $\left(\mathrm{IC}_{50}=500 \mathrm{nM}\right.$ ) kinase function (Ryan \& Wedge 2005). ZD6474 is also a potent inhibitor of the RET kinase $\left(\mathrm{IC}_{50}=100 \mathrm{nM}\right.$; Carlomagno et al. 2002). The compound could arrest the growth of human thyroid cancer cell lines spontaneously harbouring RET oncogenes and was effective against a Drosophila model of PTC and MTC (Vidal et al. 2005). The X-ray structure of ZD6474-RET(TK) complex shows that the compound docks into the ATP-binding pocket of the RET kinase (Knowles et al. 2006). In a phase II trial of hereditary MTC patients who had a germline RET mutation, ZD6474 treatment was associated with objective tumour responses, with evidence of prolonged disease stabilization and clinical benefit in up to half the patients (Wells et al. 2006). Multicentric placebocontrolled phase II trials are in progress with this drug in thyroid cancer patients (Schlumberger et al. 2008).

Preclinical in vitro studies have demonstrated that ZD6474 inhibited the wild-type enzyme and several activated mutant forms of RET, with the notable exception of RET proteins carrying mutations in residue V804 (V804L and V804M). In fact, the $\mathrm{IC}_{50}$ for RET kinase inhibition increased by about 50 -fold in the presence of V804L and V804M mutations (Carlomagno et al. 2004). V804 in RET corresponds to the 'gate-keeper' (T315) residue in ABL. V804 mutations are present alone or in conjunction with other RET mutations in MEN 2 carriers $(\sim 4 \%$ of cases) and in sporadic MTC and could therefore cause resistance to $\mathrm{ZD} 6474$.

Here, we screened natural RET oncogenic proteins, corresponding to BCR-ABL imatinib resistant mutants, for ZD6474 response in vitro and in intact cells.

\section{Materials and methods}

\section{Compound}

ZD6474 (Zactima, vandetanib; AstraZeneca Pharmaceuticals, Macclesfield, UK) was dissolved in dimethyl sulphoxide (DMSO) at a concentration of $50 \mathrm{mM}$ and 
stored at $-80{ }^{\circ} \mathrm{C} .1000 \mathrm{X}$ stock solution was freshly generated for each single experiment and the equivalent amount of vehicle (DMSO) was used as control.

\section{Cell culture and plasmids}

HEK 293 and HeLa cells were from American Type Culture Collection (Manassas, VA, USA) and were grown in DMEM supplemented with 10\% FCS, 2 mM L-glutamine and 100 units/ml penicillin-streptomycin (Gibco). Transient transfections were carried out with the lipofectamine reagent according to the manufacturer's instructions (Gibco). The alignment between RET and ABL kinase domains was made with the 'Lalign' tool available at the ExPASy proteomic tools web site (www.expasy.ch). RET mutations V804M, E805K, Y806C, Y806E, Y806F, Y806S, Y806G, $\mathrm{E} 884 \mathrm{~K}$ and D898V were generated by site-directed mutagenesis and inserted in the background of an oncogenic RET C634R allele in the pcDNA 3.1 vector (Carlomagno et al. 2004). The presence of mutation was confirmed by double-strand DNA sequencing.

\section{Protein studies}

Protein lysates were prepared according to standard procedures. Briefly, cells were lysed in a buffer

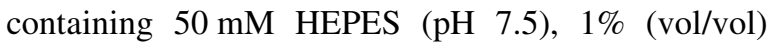
Triton X-100, $150 \mathrm{mM} \mathrm{NaCl}, 5 \mathrm{mM}$ EGTA, $50 \mathrm{mM}$ $\mathrm{NaF}, 20 \mathrm{mM}$ sodium pyrophosphate, $1 \mathrm{mM}$ sodium vanadate, $2 \mathrm{mM}$ phenylmethylsulphonyl fluoride and $1 \mu \mathrm{g} / \mathrm{ml}$ aprotinin. Lysates were clarified by centrifugation at $10000 \mathrm{~g}$ for $15 \mathrm{~min}$. Lysates containing comparable amounts of proteins, as estimated by a modified Bradford assay (Bio-Rad) were subjected to direct western blot. Immune complexes were detected with the ECL kit (Amersham Pharmacia Biotech,). Signal intensity was analysed using a Phosphorimager (Typhoon 8600, Amersham Pharmacia Biotech) interfaced with the ImageQuant software. Polyclonal anti-phospho-Shc, which recognizes phosphorylated Shc at Y317, was from Upstate Biotechnology Inc. (Lake Placid, NY, USA). Polyclonal anti-Shc was from Santa Cruz Biotechnology (Santa Cruz, CA, USA). Antibodies to MAPK were from Cell Signaling Technologies (Beverly, MA, USA), and antibodies to phospho p44/42-MAPK (pMAPK), specific for MAPK (ERK1/2) phosphorylated at Thr202/Tyr204, were from Cell Signaling Technologies. Anti-RET is a polyclonal antibody raised against the tyrosine kinase protein fragment of human RET (Santoro et al. 1994). Anti-phospho905 and anti-phospho1062 are phosphospecific polyclonal antibodies recognizing RET proteins phosphorylated at the corresponding sites (Salvatore et al. 2000, Carlomagno et al. 2003). Blots were incubated with primary antibodies for $1 \mathrm{~h}$ at room temperature, washed and incubated with the appropriate secondary antibodies (goat anti-rabbit 1:5000) coupled to HRP (Santa Cruz Biotechnology). Signal intensity was evaluated with the Molecular Imager Gel Doc System interfaced with the Quantity One software (Bio-Rad). Curves of ZD6474 inhibition were plotted to identify IC50 dose for each RET mutant in vivo autophosphorylation.

\section{In vitro kinase assay}

RET proteins were immunoprecipitated from HEK 293 cells transfected with different mutants. Immunocomplexes were subjected to an in vitro kinase assay by incubation ( $20 \mathrm{~min}$ at room temperature) in kinase buffer containing $200 \mu \mathrm{M}$ poly-(L-glutamic acid-L-tyrosine [poly-GT]; Sigma Chemical Co.), $2.5 \mu \mathrm{Ci} \gamma^{-32}$ P ATP and unlabelled ATP $(20 \mu \mathrm{M})$ and the indicated concentrations of the compound (Carlomagno et al. 2002). Samples were spotted on Whatman $3 \mathrm{MM}$ paper (Springfield Mill, UK) and ${ }^{32} \mathrm{P}$ incorporation was measured with a $\beta$-counter scintillator (Beckman Coulter, Unterschleissheim-Lohhof, Germany).

\section{Luciferase activity assay}

Approximately $1 \times 10^{6} \mathrm{HeLa}$ cells were transiently co-transfected with RET mutants and the AP1-Luc vector (Stratagene, Garden Grove, CA, USA) containing six AP1-binding sites upstream from the Firefly luciferase cDNA. Twenty-four hours after transfection, cells were serum-starved and the indicated concentration of ZD6474 or vehicle was added. Cells were harvested $48 \mathrm{~h}$ after transfection. Ten $\mathrm{ng}$ pRL-null (a plasmid expressing the enzyme Renilla luciferase from Renilla reniformis) served as an internal control. Firefly and Renilla luciferase activities were assayed using the Dual-Luciferase reporter system (Promega Corporation) and expressed as percentage of residual activity compared with cells treated with vehicle.

\section{Assessment of the structural impact of various Y806 mutations}

The structure of the RET kinase domain in complex with ZD6474 is available at Research Collaboratory for Structural Bioinformatics (RCSB) Protein Data Bank (PDB; PBD ID: 2ivu at www.rcsb.org/pdb/home/home.do; Knowles et al. 2006). Tyrosine 806 was 
replaced with the different residues and the structural consequences were examined by using the PyMOL Molecular Graphics System (http://www.pymol.org).

\section{Statistical analysis}

Kinase activity curves were plotted using the curvefitting PRIZM software (GraphPad Software). The ANOVA Post Hoc Tukey-Kramer multiple comparison test was used to assess statistical significance of luciferase assay. InStat3 GraphPad Software was used.

\section{Results}

\section{Identification of Y806 as a molecular determinant of RET sensitivity to ZD6474 inhibition}

We aligned the RET and ABL kinase domains and found that several RET residues, naturally mutated in some MEN 2/MTC patients, correspond to positions in $\mathrm{ABL}$ whose mutation causes imatinib resistance (Fig. 1A). RET changes in E768, L790 and A883 residues (corresponding to E279, L301 and R372 in ABL respectively) do not affect RET kinase sensitivity to ZD6474, as previously reported (Carlomagno et al. 2004). Here, we evaluated the effect of $\mathrm{E} 805 \mathrm{~K}$, Y806C, E884K and D898V (corresponding to the imatinib-resistant E316D, ABL F317L, E373K and L387M mutants respectively) on RET kinase sensitivity to ZD6474 (Fig. 1A). The E805K mutation was found in tandem with V804M in a patient with a MEN 2B phenotype (Cranston et al. 2006). The adjacent Y806 residue was found mutated to cysteine (Y806C) at the germline level in combination with mutation V804 in a patient with MEN 2B-like clinical presentation (Miyauchi et al. 1999, Iwashita et al. 2000). Notably, phospho-amino acid analysis included Y806 among RET kinase autophosphorylation sites (Kawamoto et al. 2004). Both E805 and Y806 map in the RET catalytic domain to the same loop containing the gate-keeper V804 residue (Knowles et al. 2006). Moreover, the E884K mutation was identified in individual sporadic MTC case (Uchino et al. 1999). Finally, residuze D898 has been found deleted in a sporadic MTC patient. The same aspartic acid in KIT kinase was shown to mediate resistance to imatinib when mutated to valine and ABL L387 residue, located in the corresponding position, was mutated in an imatinib-resistant kinase (Oriola et al. 2002).

Because these mutations weakly activate RET kinase (Miyauchi et al. 1999, Iwashita et al. 2000), we introduced them in the background of a

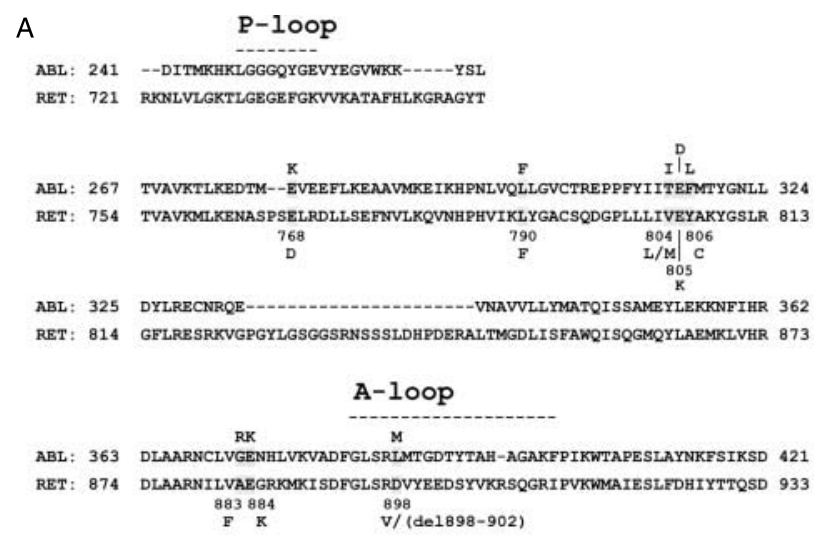

B
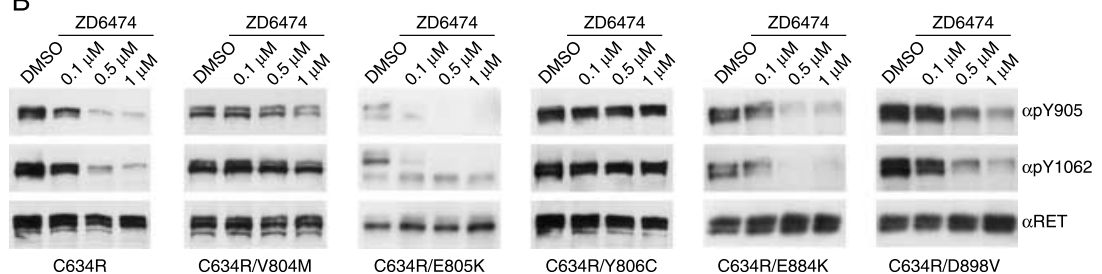

Figure 1 Y806 residue determines RET kinase sensitivity to ZD6474. (A) Alignment of ABL and RET kinase domains. P-Loop (phosphate-binding loop) and A-loop (activation loop) are indicated. Mutations that cause resistance to imatinib in ABL and that correspond to naturally occurring RET mutations are highlighted in grey. For RET, the position of the mutated amino acid is reported. (B) HEK293 cells were transfected with pcDNA 3.1 vectors expressing the indicated RET mutants. Twenty-four hours after transfection, cells were serum-starved. Two hours before harvest, cells were treated with various ZD6474 doses or vehicle. Cell lysates were immunoblotted with the indicated antibodies. Each immunoblot is representative of at least three independent experiments. 
constitutively active RET allele carrying the MEN 2Aassociated extracellular C634R mutation. C634R strongly activates RET by inducing a constitutive dimerization of the receptor through the formation of extracellular disulphide bonds without affecting the natural conformation of RET kinase domain (Santoro \& Carlomagno 2006). Intact cells expressing RET mutants were treated with different ZD6474 doses and RET activity was measured by immunoblotting with anti-phospho RET antibodies that recognize the Y905 and Y1062 autophosphorylation sites (Salvatore et al. 2000, Carlomagno et al. 2003). When phosphorylated, Y1062 functions as multidocking site for intracellular signalling proteins, whereas Y905 resides in the activation loop and its phosphorylation is necessary to maintain the kinase in an active conformation (Hayashi et al. 2000, Iwashita et al. 2000). As shown in Fig. 1B, ZD6474 inhibited RET proteins carrying C634R/E805K $(\mathrm{IC} 50=100 \mathrm{nM}), \mathrm{C} 634 \mathrm{R} / \mathrm{E} 884 \mathrm{~K}(\mathrm{IC} 50=200 \mathrm{nM})$ or C634R/D898V (IC50 $=200 \mathrm{nM}$ ) tandem substitutions similarly to RET C634R protein $(\mathrm{IC} 50=100 \mathrm{nM})$. By contrast, the RET C634R/Y806C protein was highly resistant to the drug (IC50>1000 nM). The ZD6474resistant RET C634R/V804M mutant served as control (IC50>1000 nM; Fig. 1B).

\section{Structure-function analysis of the effect of RET Y806 mutation}

We generated RET mutants carrying mutations of Y806 to different amino acids, namely glycine (Y806G), serine (Y806S), glutamic acid (Y806E) and phenylalanine $(\mathrm{Y} 806 \mathrm{~F})$, in the background of RET C634R. Mutations Y806G and Y806S knocked down kinase activity (not shown) and were not studied further. The substitution of tyrosine 806 with the nonphosphorylatable phenylalanine (Y806F) did not significantly affect kinase activity. In addition, it did not modify RET sensitivity to ZD6474, which suggested that Y806 phosphorylation was not necessary for ZD6474 binding (Fig. 2A). By contrast, the substitution of tyrosine 806 with the phosphomimetic glutamate (Y806E) greatly reduced RET sensitivity to ZD6474, and thus had an effect comparable with the Y806C change (Fig. 2A).

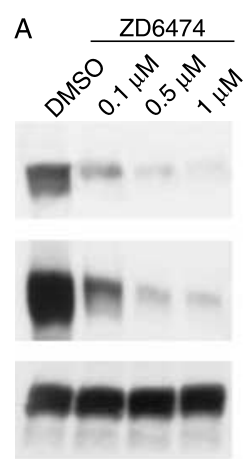

C634R

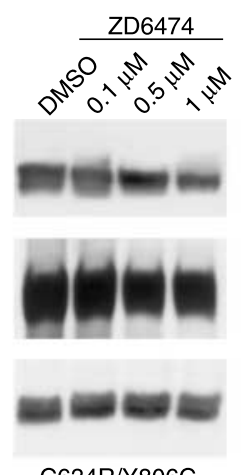

C634R/Y806C

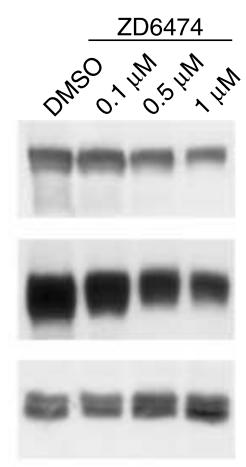

C634R/Y806E

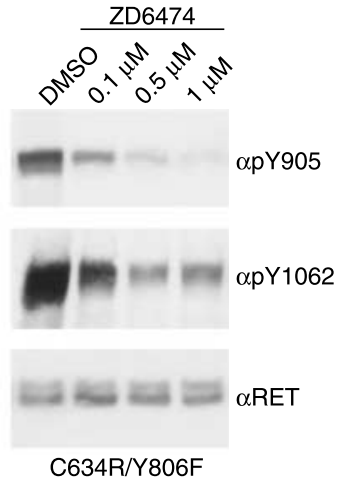

$\mathrm{C} 634 \mathrm{R} / \mathrm{Y} 806 \mathrm{~F}$

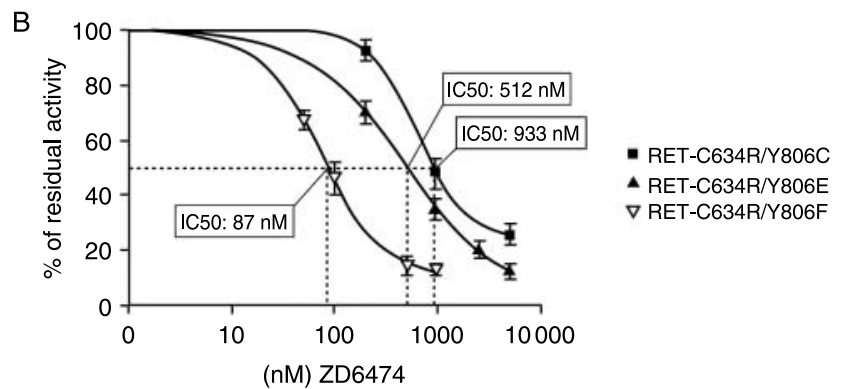

Figure 2 Effect of alternative RET Y806 substitutions on sensitivity to ZD6474. (A) HEK293 cells were transfected with pcDNA 3.1 vectors expressing the indicated RET mutants. Twenty-four hours after transfection, cells were serum-starved and treated $(2 \mathrm{~h})$ with ZD6474 or vehicle. Cell lysates were analysed by immunoblot with the indicated antibodies. Each immunoblot is representative of at least three independent experiments. (B) HEK293 cells were transfected with RET mutants-expressing vectors. RET proteins were immunoprecipitated and subjected to an in vitro kinase assay on an artificial substrate (poly-Glu-Tyr). Each experiment was performed in triplicate and done at least thrice. Results from kinase assay have been plotted using a curve-fitting software. S.D. are indicated. 
Subsequently, we carried out an in vitro kinase assay and measured ZD6474 in vitro $\mathrm{IC}_{50}$ for the various RET Y806 mutants. As shown in Fig. 2B, ZD6474 $\mathrm{IC}_{50}$ for the RET C634R/Y806F protein was similar to RET C634R (87 nM vs $100 \mathrm{nM}$ ), whereas the $\mathrm{IC}_{50}$ values for RET C634R/Y806E and RET C634R/Y806C were increased by fivefold $(512 \mathrm{nM})$ and tenfold $(933 \mathrm{nM})$ respectively.

\section{Analysis of ZD6474 activity on intracellular signalling of RET Y806 mutants}

RET phosphorylation on Y1062 results in a docking site for Shc and other adaptor proteins that, in turn, serve as anchors for Grb2/Sos and Grb2/Gab complexes thereby mediating RET signals to downstream pathways such as the PI3K/AKT and RAS/MAPK ones (Hayashi et al. 2000). Similar to RET C634R/V804M, phosphorylation of SHC by RET C634R/Y806C and RET C634R/ Y806E, as well as MAPK (ERK1/2) activation, showed resistance to ZD6474 (Fig. 3A).

Finally, we tested whether ZD6474 obstructed RETmediated activation of an AP1-responsive promoter fused to the luciferase reporter. As shown in Fig. 3B, the compound reduced RET C634R activity to less than $50 \%$ at $250 \mathrm{nM}$ and completely abolished promoter activation at $1 \mu \mathrm{M}$. Instead, ZD6474, even at a concentration of $5 \mu \mathrm{M}$, had virtually no effect on RET C634R/V804M-mediated luciferase stimulation. Compared with RET C634R, RET mutants C634R/Y806C and RET C634R/Y806E were also relatively resistant to the effects of ZD6474, although to a lesser extent than RET C634R/V804M.

\section{Discussion}

The efficacy of ZD6474 in targeting RET oncogenic mutations in MTC is now being evaluated in phase II clinical trials. Although clinical benefit from ZD6474 is likely to derive from an inhibition of both VEGFR-2 and EGFR kinase activities in addition to RET kinase, it will be necessary to identify RET mutations that respond best to the compound in order to interpret the results of these trials and subsequently to select the most suitable candidates for ZD6474 treatment. Here, we screened natural oncogenic RET kinase mutants for ZD6474 response. The majority of these mutants, although homologous to ABL mutants resistant to imatinib, retain ZD6474 responsiveness. An important
A

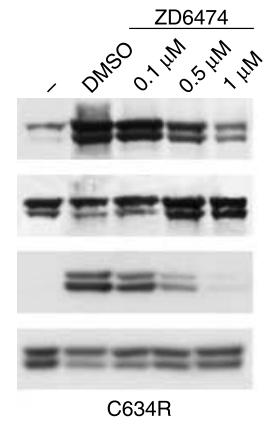

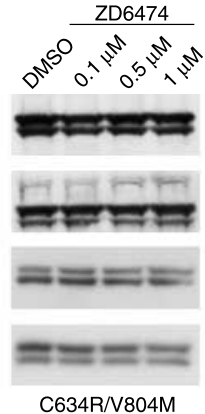

C634R/V804M

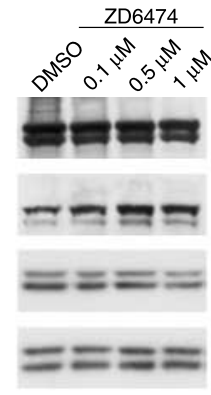

C634R/v806C

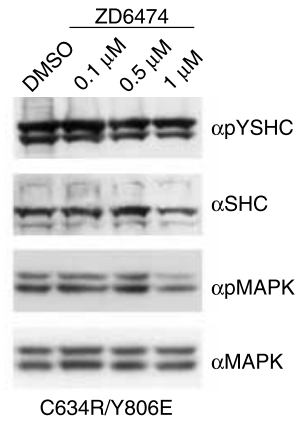

$\mathrm{C} 634 \mathrm{R} / \mathrm{Y} 806 \mathrm{E}$

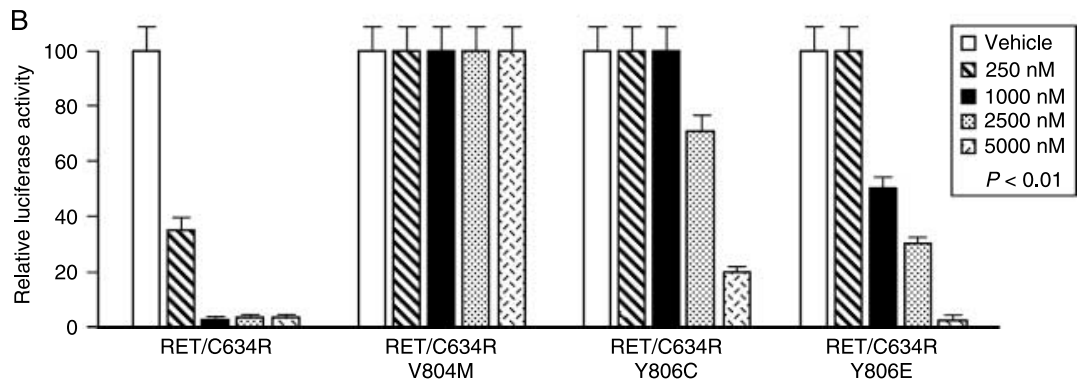

Figure 3 ZD6474 effects on RET mutants intracellular signalling. (A) HEK293 cells were transfected with RET mutants expressing vectors. Twenty-four hours after transfection, cells were serum-starved and treated $(2 \mathrm{~h})$ with the indicated concentration of ZD6474. Cell lysates were analysed by immunoblot with the indicated antibodies. Each experiment is representative of at least three assays. (B) Approximately $1 \times 10^{6}$ HeLa cells were transiently transfected with RET mutants expressing vectors and the AP1-Luc vector pRL-null (a plasmid expressing the enzyme Renilla luciferase from Renilla reniformis) served as an internal control. Firefly and Renilla luciferase activities are expressed as percentage of residual activity compared with the cells treated with vehicle. Average results of three independent assays \pm s.D. are indicated. The ANOVA Post Hoc Tukey-Kramer multiple comparison test was used to demonstrate statistical significance $(P<0.01)$. 
A

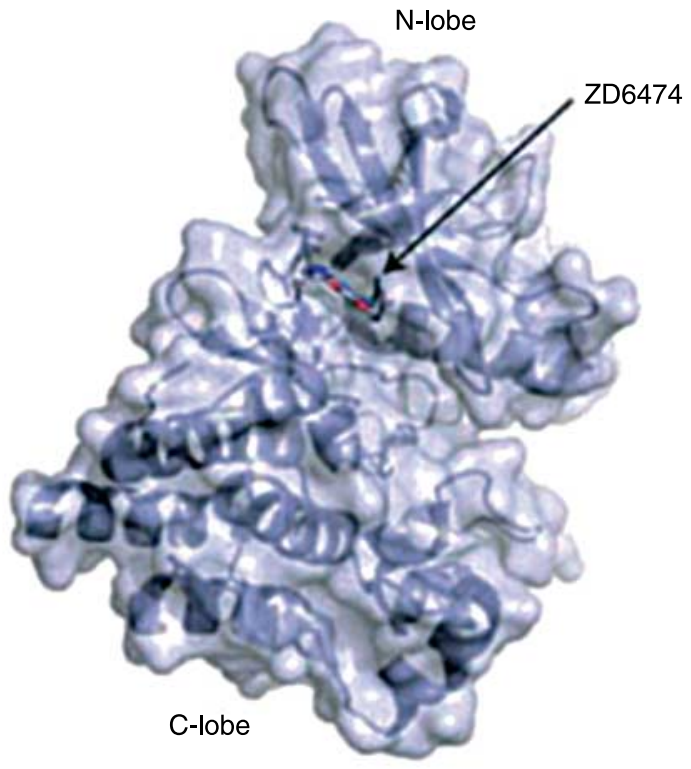

B

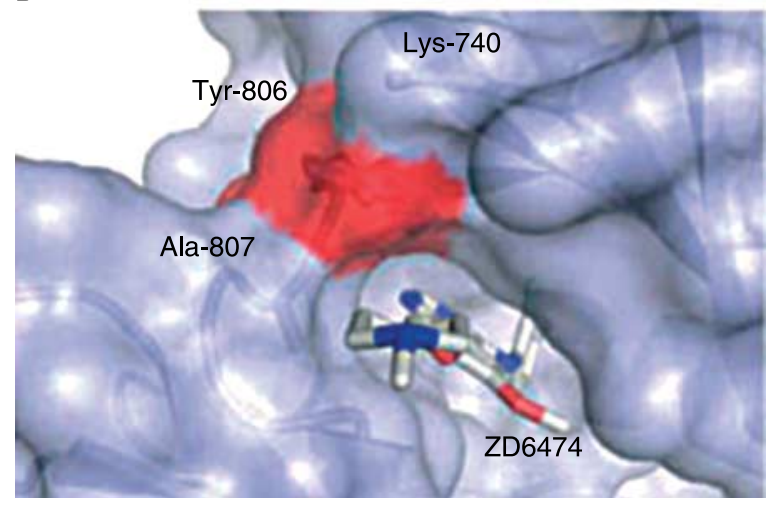

Figure 4 Structure of RET Kinase-ZD6474 complex. (A) The kinase domain of RET in complex with ZD6474. ZD6474 binds in the nucleotide-binding pocket between the $\mathrm{N}$ - and $\mathrm{C}$-lobes.

(B) Close-up of the nucleotide-binding pocket showing how the tyrosine 806 (red) interacts with the aliphatic chain of lysine 740 and the main chain moiety of alanine 807 , and establishes Van der Waals contacts with ZD6474.

exception is Y806C that, similar to V804M/L (Carlomagno et al. 2004), impairs ZD6474 efficacy.

Based on the recently resolved RET kinase X-ray structure (Knowles et al. 2006), tyrosine 806 is located between the kinase $\mathrm{N}$ - and $\mathrm{C}$-lobes in the hinge region that forms part of the nucleotide-binding pocket (Fig. 4A). The Y806 side chain stacks against the aliphatic part of the lysine 740 (K740) side chain and the main chain of the adjacent alanine 807 (A807; Fig. 4B). The position equivalent to Y806 in other kinases is usually hydrophobic and aromatic, which might explain why we found that substitution of this residue with glycine or serine dramatically affects kinase function. Y806 is in direct Van der Waals contact with ATP and ZD6474. Conservative substitution of Y806 to phenylalanine (Y806F) preserves the shape and hydrophobicity of this part of the nucleotide pocket. Therefore, this mutated RET is likely to maintain contacts with ZD6474 using Y806, which explains why the Y806F mutation does not affect the sensitivity of RET towards ZD6474. On the other hand, the effects of the Y806C and Y806E substitutions are subtle and unpredictable compared with the V804M/L mutation, which causes a clear steric hindrance (Knowles et al. 2006). Replacement of Y806 by a smaller cysteine will leave a cavity thereby leading to minor structural alterations to the nucleotide-binding pocket, so that the kinase retains the ability to bind and hydrolyse ATP. On the other hand, these changes could affect affinity for ZD6474 by losing direct Van der Vaals interactions. Substitution of Y806 with the acidic glutamate side chain introduces a negative charge into the hydrophobic environment of the inhibitor-binding site. This may lead to an interaction with the adjacent accessible K740 side chain and, in turn, perturb the alignment of the hinge region. Differences in the hinge region are indeed seen in RET structures bound to ATP compared with ZD6474 (Knowles et al. 2006). A possible consequence of Y806E would be an altered E805 conformation. The main chain carbonyl of E805 makes direct contact with ZD6474, and loss of this interaction may impact on inhibitor sensitivity. This may also explain why replacing the E805 side chain by lysine (E805K) appears not to affect the actions of ZD6474.

In conclusion, Y806 is involved in RET response to ZD6474. Y806 mutation may be an extremely rare primary mutation causing ZD6474 refractoriness. However, mutations at Y806 and Y806-containing loop including V804, but not at E805, may mediate secondary resistance during the treatment of thyroid cancer patients with ZD6474. This information would be useful in investigating the mechanisms of lack of response to ZD6474 treatment and designing secondline inhibitors to overcome resistance.

\section{Declaration of interest}

A J Ryan is a full-time employee of Astra Zeneca. M Santoro received an Astra Zeneca research grant.

\section{Funding}

This study was supported by the Associazione Italiana per la Ricerca sul Cancro (AIRC), the NOGEC (Naples OncoGEnomic Center), by grants from Italian Ministero della Salute and Ministero dell'Università e della Ricerca and by 
a research grant from Astra Zeneca. T Guida was supported by an AIRC fellowship. S Anaganti was supported by a Terry Fox Foundation fellowship.

\section{Acknowledgements}

We thank Jean Ann Gilder for text editing and Ciotola Presentation for the art work.

\section{References}

Baselga J 2006 Targeting tyrosine kinases in cancer: the second wave. Science 312 1175-1178.

Bell DW, Gore I, Okimoto RA, Godin-Heymann N, Sordella R, Mulloy R, Sharma SV, Brannigan BW, Mohapatra G, Settleman J et al. 2005 Inherited susceptibility to lung cancer may be associated with the T790M drug resistance mutation in EGFR. Nature Genetics 37 1315-1316.

Carlomagno F, Vitagliano D, Guida T, Ciardiello F, Tortora G, Vecchio G, Ryan AJ, Fontanini G, Fusco A \& Santoro M 2002 ZD6474, an orally available inhibitor of KDR tyrosine kinase activity, efficiently blocks oncogenic RET kinases. Cancer Research 62 7284-7290.

Carlomagno F, Vitagliano D, Guida T, Basolo F, Castellone MD, Melillo RM, Fusco A \& Santoro M 2003 Efficient inhibition of RET/papillary thyroid carcinoma oncogenic kinases by 4-amino-5-(4-chloro-phenyl)-7-(t-butyl)pyrazolo[3,4-d]pyrimidine (PP2). Journal of Clinical Endocrinology and Metabolism 88 1897-1902.

Carlomagno F, Guida T, Anaganti S, Vecchio G, Fusco A, Ryan AJ, Billaud M \& Santoro M 2004 Disease associated mutations at valine 804 in the RET receptor tyrosine kinase confer resistance to selective kinase inhibitors. Oncogene 23 6056-6063.

Corless CL, Schroeder A, Griffith D, Town A, McGreevey L, Harrell P, Shiraga S, Bainbridge T, Morich J \& Heinrich MC 2005 PDGFRA mutations in gastrointestinal stromal tumors: frequency, spectrum and in vitro sensitivity to imatinib. Journal of Clinical Oncology 23 5357-5364.

Cranston AN, Carniti C, Oakhill K, Radzio-Andzelm E, Stone EA, McCallion AS, Hodgson S, Clarke S, Mondellini P, Leyland J et al. 2006 RET is constitutively activated by novel tandem mutations that alter the active site resulting in multiple endocrine neoplasia type $2 \mathrm{~B}$. Cancer Research 66 10179-10187.

Daub H, Specht K \& Ullrich A 2004 Strategies to overcome resistance to targeted protein kinase inhibitors. Nature Reviews. Drug Discovery 3 1001-1010.

Hayashi H, Ichihara M, Iwashita T, Murakami H, Shimono Y, Kawai K, Kurokawa K, Murakumo Y, Imai T, Funahashi H et al. 2000 Characterization of intracellular signals via tyrosine 1062 in RET activated by glial cell line-derived neurotrophic factor. Oncogene 19 4469-4475.

Iwashita T, Murakami H, Kurokawa K, Kawai K, Miyauchi A, Futami H, Qiao S, Ichihara M \& Takahashi M 2000 A two-hit model for development of multiple endocrine neoplasia type 2B by RET mutations. Biochemical and Biophysical Research Communications 268 804-808.
Kantarjian H, Giles F, Wunderle L, Bhalla K, O'Brien S, Wassmann B, Tanaka C, Manley P, Rae P, Mietlowski W et al. 2006 Nilotinib in imatinib-resistant CML and Philadelphia chromosome-positive ALL. New England Journal of Medicine 354 2542-2551.

Kawamoto Y, Takeda K, Okuno Y, Yamakawa Y, Ito Y, Taguchi R, Kato M, Suzuki H, Takahashi M \& Nakashima I 2004 Identification of RET autophosphorylation sites by mass spectrometry. Journal of Biological Chemistry 279 14213-14224.

Knowles PP, Murray-Rust J, Kjaer S, Scott RP, Hanrahan S, Santoro M, Ibáñez CF \& McDonald NQ 2006 Structure and chemical inhibition of the RET tyrosine kinase domain. Journal of Biological Chemistry 281 33577-33587.

Krause DS \& Van Etten RA 2005 Tyrosine kinases as targets for cancer therapy. New England Journal of Medicine $\mathbf{3 5 3}$ 172-187.

Marx SJ 2005 Molecular genetics of multiple endocrine neoplasia types 1 and 2. Nature Reviews. Cancer $\mathbf{5}$ 367-375.

Miyauchi A, Futami H, Hai N, Yokozawa T, Kuma K, Aoki N, Kosugi S, Sugano K \& Yamaguchi K 1999 Two germline missense mutations at codons 804 and 806 of the RET proto-oncogene in the same allele in a patient with multiple endocrine neoplasia type 2B without codon 918 mutation. Japanese Journal of Cancer Research 90 1-5.

Nardi V, Azam M \& Daley GQ 2004 Mechanisms and implications of Imatinib resistance mutations in BCRABL. Current Opinion in Hematology 11 35-43.

O'Hare T, Corbin AS \& Druker BJ 2006 Targeted CML therapy: controlling drug resistance, seeking cure. Current Opinion in Genetics \& Development 16 92-99.

Oriola J, Halperin I, Rivera-Fillat F \& Donis-Keller H 2002 The finding of a somatic deletion in RET exon 15 clarified the sporadic nature of a medullary thyroid carcinoma suspected to be familial. Journal of Endocrinological Investigation 25 25-31.

Pao W, Miller VA, Politi KA, Riely GJ, Somwar R, Zakowski MF, Kris MG \& Varmus H 2005 Acquired resistance of lung adenocarcinomas to gefitinib or erlotinib is associated with a second mutation in the EGFR kinase domain. PLoS Medicine 2 e73.

Ryan AJ \& Wedge SR 2005 ZD6474 - a novel inhibitor of VEGFR and EGFR tyrosine kinase activity. British Journal of Cancer 92 S6-S13.

Salvatore D, Barone MV, Salvatore G, Melillo RM, Chiappetta G, Mineo A, Fenzi G, Vecchio G, Fusco A \& Santoro M 2000 Tyrosines 1015 and 1062 are in vivo autophosphorylation sites in ret and ret-derived oncoproteins. Journal of Clinical Endocrinology and Metabolism 85 3898-3907.

Santoro M \& Carlomagno F 2006 Drug insight: smallmolecule inhibitors of protein kinases in the treatment of thyroid cancer. Nature Clinical Practice. Endocrinology \& Metabolism 2 42-52.

Santoro M, Wong WT, Aroca P, Santos E, Matoskova B, Grieco M, Fusco A \& di Fiore PP 1994 An epidermal 
growth factor receptor/ret chimera generates mitogenic and transforming signals: evidence for a ret-specific signaling pathway. Molecular and Cellular Biology 14 663-675.

Schlumberger M, Carlomagno F, Baudin E, Bidart JM \& Santoro M 2008 New therapeutic approaches to treat medullary thyroid carcinoma. Nature Clinical Practice. Endocrinology \& Metabolism 4 22-32.

Shah NP, Skaggs BJ, Branford S, Hughes TP, Nicoll JM, Paquette RL \& Sawyers CL 2007 Sequential ABL kinase inhibitor therapy selects for compound drugresistant BCR-ABL mutations with altered oncogenic potency. Journal of Clinical Investigation 117 2562-2569.

Sherbenou DW \& Druker BJ 2007 Applying the discovery of the Philadelphia chromosome. Journal of Clinical Investigation 117 2067-2074.

Talpaz M, Shah NP, Kantarjian H, Donato N, Nicoll J, Paquette R, Cortes J, O'Brien S, Nicaise C, Bleickardt E et al. 2006 Dasatinib in Imatinib-resistant Philadelphia chromosome-positive leukemias. New England Journal of Medicine 354 2531-2541.
Uchino S, Noguchi S, Yamashita H, Sato M, Adachi M, Yamashita H, Watanabe S, Ohshima A, Mitsuyama S, Iwashita T et al. 1999 Somatic mutations in RET exons 12 and 15 in sporadic medullary thyroid carcinomas: different spectrum of mutations in sporadic type from hereditary type. Japanese Journal of Cancer Research 90 1231-1237.

Vidal M, Wells S, Ryan A \& Cagan R 2005 ZD6474 suppresses oncogenic RET isoforms in a Drosophila model for type 2 multiple endocrine neoplasia syndromes and papillary thyroid carcinoma. Cancer Research $\mathbf{6 5}$ 3538-3541.

Weisberg E, Manley PW, Cowan-Jacob SW, Hochhaus A \& Griffin JD 2007 Second generation inhibitors of BCRABL for the treatment of Imatinib-resistant chronic myeloid leukaemia. Nature Reviews. Cancer 7 345-356.

Wells S, You YN, Lakhani V, Hou J, Langmuir P, Headley D, Skinner M, Morse M, Burch W \& Schlumberger M 2006 A phase II trial of ZD6474 in patients with hereditary metastatic medullary thyroid cancer. Journal of Clinical Oncology 245533 (ASCO Annual Meeting Proceedings Part I). 